\title{
Untangling Triple-Negative Breast Cancer Molecular Peculiarity and Chemo-Resistance: Trailing Towards Marker-Based Targeted Therapies
}

Bushra Kanwal 1, 2

1. Internal Medicine, Brookdale University Hospital Medical Center, Brooklyn, USA 2. Lombardi Comprehensive Cancer Center, Georgetown University Medical Center, Washington, D.C., USA

Corresponding author: Bushra Kanwal, bushrafass@yahoo.com

\begin{abstract}
Triple-negative breast cancer (TNBC), characterized by the absence of estrogen receptor, progesterone receptor, or human epidermal growth factor receptor- 2 , affects nearly $15 \%$ of women with breast cancer. To date, the mainstay of treatment remains chemotherapy, with all the associated consequences, such as the significant toxicity and the suboptimal effect on the five-year survival rates. RNA-expression profiling showed that TNBC is biologically a heterogeneous malignancy. Therefore, predictive biomarkers matched with the diverse subtypes of TNBC could classify patients that would most benefit from a certain targeted treatment. Three biomarker-driven therapies are currently available: poly-adenosine diphosphate (ADP) ribose polymerase inhibitors for patients with germline BReast CAncer gene (BRCA) mutations, atezolizumab combined with nab-paclitaxel for patients expressing programmed death-ligand 1 (PD-L1) on tumor-infiltrating immune cells, and sacituzumab govitecan, an antibody-drug conjugate targeting human trophoblast cell-surface antigen 2 (TROP-2). Identifying predictive biomarkers is crucial for the optimum generation and implementation of targeted agents for TNBC, while further relevant treatments are in the pipeline given the promising results in clinical trials. Finally, newly developed immunotherapies and other targeted agents should also be investigated in earlier stages of the disease, especially in the neoadjuvant setting, broadening the therapeutic application of such regimens.
\end{abstract}

Review began $07 / 08 / 2021$ Review ended 07/18/2021 Published 07/26/2021

○) Copyright 2021 Kanwal. This is an open access article distributed under the terms of the Creative Commons Attribution License CC-BY 4.0., which permits unrestricted use, distribution, and reproduction in any medium, provided the original author and source are credited.
Categories: Internal Medicine, Oncology, Healthcare Technology

Keywords: triple-negative breast cancer, estrogen receptor, progesterone receptor, biomarker, brca1, immune checkpoint inhibitors, human epidermal growth factor receptor-2, parp inhibitors, breast cancer, rna-expression profiling

\section{Introduction And Background}

Breast cancer is the most prevalent malignancy in females worldwide, while metastatic breast cancers are the second leading cause of tumor-related deaths in American women. Only in the United States, more than 3.5 million women have been diagnosed with breast cancer [1]. In 2021, it is estimated that approximately 280,000 new cases of invasive breast cancer will be diagnosed in the United States, and about 43,600 deaths will occur [2].

Almost one out of five cases of breast cancer are identified as triple-negative breast cancer (TNBC). This type is characterized by the absence of estrogen and progesterone receptors and the lack of human epidermal growth factor receptor-2 amplification. Unlike hormone receptor-positive or human epidermal growth factor receptor (HER) 2 positive breast cancer, TNBC affects mainly young (age < 50 years) AfricanAmerican women, especially those who carry the BReast CAncer gene (BRCA) 1/2 mutations [3]. This type of breast cancer is particularly aggressive with high mortality, frequent relapses, and extensive metastases. Nevertheless, the management of TNBC is challenging due to its resistance to typical therapies for other types of breast cancer, such as hormonal therapy and HER-2 antagonists treatments [4].

Even with substantial toxicity, frequent relapses, and low survival rates, chemotherapy remains the primary therapeutic approach for triple-negative breast cancer (TNBC). Based on the relevant transcriptomic analyses, TNBC is a biologically heterogeneous tumor with diverse molecular and phenotypical features. This analysis insight to customized two-step approach of first identifying the patient's TNBC subtype with its responsible predictive biomarkers and rendering with designed targeted therapy.

The biomarker-guided therapies have recently been approved, including poly-ADP ribose polymerase inhibitors for patients with germline BRCA mutations and atezolizumab in conjunction with nab-paclitaxel for patients expressing programmed death-ligand 1 (PD-L1) on tumor-infiltrating immune cells. Furthermore, the newly developed antibody-drug conjugate sacituzumab govitecan-hziy is recently approved for cases with metastatic TNBC, which have progressed on at least two previous lines of treatment for advanced disease $[5,6]$. 
The identification of specific biomarkers which would facilitate customized treatment decision-making in TNBC has not yet been achieved. Therefore, this review aimed to explore the molecular heterogeneity of TNBC, study the mechanisms of chemo-resistance, and present potential biomarkers for the generation of targeted therapies. By this means, the objective of such treatments is to prevent the development of metastatic disease and, eventually, to increase the survival rates.

\section{Review}

\section{Triple-negative breast cancer}

Breast cancer is one of the most common tumors among women demonstrating high mortality rates [7]. TNBC is defined as the subtype of breast cancer and accounts for nearly $20 \%$ of diagnosed cases of breast cancers, affecting primarily younger women. Techniques like gene expression and DNA sequencing are employed to classify TNBC [8]. It is one of the most aggressive types linked to high recurrence, metastatic rates, and poor overall survival [9].

\section{Molecular peculiarity - molecular classification upon genomic profiling}

Typical subclassification of invasive ductal breast cancers is obtained by immunostaining tumor tissues for estrogen receptor (ER), progesterone receptor (PR), HER-1, and HER-2, and various cytokeratins. An immunohistochemical staining proxy based on biomarkers categorizes breast cancer into five significant subtypes: (1) luminal A (ER-positive and/or PR positive, HER-2 negative); (2) luminal B (ER-positive and/or PR positive, HER-2 positive); (3) HER-2 overexpressing (ER-negative, PR negative, HER-2 positive); (4) basal-like (ER-negative, PR negative, HER-2 negative, cytokeratin 5/6 positive and/or epidermal growth factor receptor $\{E G F R\}$ positive); and (5) normal breast-like tumors (unclassified) were negative for all five biomarkers. Basal-like breast cancer is differentiated from other TNBC (ER, PR, and HER-2 negative) by expressing cytokeratin 5/6 and/or EGFR. Triple-negative breast cancer is diagnosed through immunohistochemistry (IHC). Nevertheless, the diagnosis is challenging due to the molecular overlap between the triple-negative phenotype and the basal-like subtype of breast cancer (Table 1). Because basallike breast cancers are usually triple-negative, there is a mistaken belief that these two terms are synonymous. Yet, $70-80 \%$ of TNBC are basal-like, whereas approximately $70 \%$ of basal-like tumors are triplenegative $[8,10,11]$.

\begin{tabular}{|c|c|c|c|c|c|}
\hline Molecular Subtypes & ER & PR & HER-2 & Cytokeratin 5 and 6 & Prevalence \\
\hline Luminal A & Positive & Positive & Negative & Negatıve & $\sim 40 \%$ \\
\hline Lumınal B & Positive & Positive & Pos/Neg & Negative & $\sim 20 \%$ \\
\hline HER-2 enriched & Negative & Negative & Positive & Negative & $\sim 10-15 \%$ \\
\hline Basal-like & Negative & Negative & Negative & Positive & $\sim 15-20 \%$ \\
\hline Unclassified & Negative & Negative & Negative & Negative & $\sim 2-8 \%$ \\
\hline
\end{tabular}

TABLE 1: Characteristics of molecular subtypes of breast cancer

Pos/Neg: positive/negative; HER-2: human epidermal growth factor receptor 2; ER: estrogen receptor; PR: progesterone receptor

Treatment challenges - a deviation from novel breast cancer therapy

Like almost any epithelial cancer, breast cancer correlates with more favorable therapeutic and survival outcomes when detected early. Nevertheless, even if breast cancer is diagnosed early, the outcomes may vary across the different molecular subtypes. Usually, the ER-positive breast cancer subtypes, such as luminal A and luminal B, demonstrate a good prognosis with optimal five-year survival rates ranging between $80 \%$ and $85 \%$, while the ER-negative subtypes (HER-2-positive and basal-like) are more challenging in their treatment and are linked to poor prognosis with a 50-60\% five-year survival rate (Table 1). This variance of outcomes could be partially attributed to the different responses of ER-positive subtypes to antiestrogenic regimens. Targeted treatment of HER-2 overexpressing breast cancers, like luminal B or HER-2positive/ER-negative carcinomas, with trastuzumab (Herceptin), either concurrent or sequential with adjuvant chemotherapy, has enhanced survival for patients with these breast cancer subtypes $[12,13]$.

TNBC is characterized by an autonomous growth independently of the expression of hormone receptors. Given, thus, the absence of those receptors, medications like tamoxifen (targeting ER) and trastuzumab (targeting HER-2) do not affect patients with this type of breast cancer. Their variable response to chemotherapy and the high incidence of recurrences justifies the fact that TNBCs demonstrate poor clinical outcomes, overall poor prognosis, and shorter long-term survival. Therapeutic resistance coupled with the 
lack of proper molecular targets and poor prognosis makes detecting biomarkers and relevant therapeutic targets in triple-negative breast cancers of utmost importance [14,15].

\section{TNBC chemotherapy}

Chemotherapy is the first-line treatment for TNBC in the neoadjuvant, adjuvant, or metastatic setting. Taxane and anthracycline-based therapies constitute the primary therapeutic option in TNBC management, whereas platinum-based chemotherapy may also play a role in neoadjuvant and metastatic cases. Although TNBC may be aggressive, up to $40 \%$ of patients obtain a complete clinical response (CR) with the concurrent histopathological response at the time of surgery after neoadjuvant chemotherapy, exhibiting higher survival rates. Nevertheless, patients with residual malignant tissues after neoadjuvant chemotherapy have a six-fold likelihood to experience a relapse and a twelve-fold metastatic mortality rate [16].

\section{TNBC chemoresistance}

Although neoadjuvant chemotherapy is beneficial for patients with TNBC, almost half of them may develop resistance and, consequently, poor overall survival. The genomic and molecular origin of this resistance of TNBC remains unclear, partly because there is still a lack of efficient techniques that can determine the intratumor heterogeneity and identify genomic information in unique subpopulations [17,18].

Previous studies on the chemo-resistance of TNBC have concentrated chiefly on in situ hybridization techniques and bulk genomic profiling procedures [19,20]. Using targeted cytogenetic biomarkers, research has shown that genetic diversity did not alter in response to neoadjuvant chemotherapy; on the contrary, it appeared selected for mesenchymal phenotypes [19]. Studies were conducted utilizing next-generation sequencing techniques to profile post-chemotherapy resistant TNBC samples to recognize several clinically meaningful mutations [20]. Single-cell DNA and RNA sequencing procedures can function as valuable tools for resolving intratumor heterogeneity, reforming evolutionary lineages, and identifying uncommon subpopulations [21,22].

\section{Biomarker-based targeted therapies}

Chemoresistant TNBC demonstrates genetic diversity, high heterogeneity, and rapid progression, making customized treatment for incomplete responders and relapsed cases a daunting task. Several FDA-approved regimens targeting programmed cell death protein-1 (pembrolizumab) and programmed death ligand-1 (atezolizumab), poly adenosine diphosphate (ADP)-ribose polymerase (olaparib and talazoparib), and/or antibody-drug conjugates (sacituzumab govitecan) have provided promising clinical results for specific patients with TNBC. These inhibitors targeting key genetic mutations and certain molecular signaling pathways that control carcinoma growth have been employed as single agents and/or in conjunction with typical chemotherapy drugs [23]. Next, we present the biomarkers that were involved in the development of novel approved treatments in TNBC.

\section{BRCA1 and BRCA2 genes - PARP inhibitors}

Compared to other subtypes of breast cancer, patients with TNBC show a significant prevalence of germline BRCA mutations (gBRCAm), reaching up to 31\% [24]. BRCA1 and BRCA2 are autosomal dominant and tumor suppressor genes that preserve genome integrity. Both genes are involved in the homologous recombination repair (HRR) of DNA. In cells with BRCA1/2 deficiency, DNA double-strand breaks (DSBs) repair depend on poly adenosine diphosphate-ribose polymerase (PARP)1 protein [25]. PARP is an abundant, constitutively expressed nuclear enzyme that promotes DNA repair, cellular proliferation, and signaling to other critical cell-cycle proteins and oncogenes. Wherever there is DNA damage, PARP stimulates intracellular signaling pathways that regulate DNA repair and, therefore, cell survival [26]. As a consequence, inhibition of PARP1 coupled with BRCA1 and BRCA2 deficiency may result in serious, highly selective toxicity in these tumor cells [27].

Lately, PARP inhibitors have been comprehensively investigated as a targeted treatment for geminal BRCA mutations (gBRCAm) in ovarian and breast cancer. PARP inhibitors, such as olaparib, under the brand name Lynparza, and talazoparib, under the brand name Talzenna, have been approved for the management of advanced HER-2-negative breast cancer in individuals with a BRCA1 or BRCA2 mutation [28].

In January 2018, based on the beneficial outcomes from the OlympiAD trial, the FDA approved olaparib for the treatment of cases with deleterious or potentially deleterious gBRCAm, HER-2-negative metastatic breast cancer which failed neoadjuvant, adjuvant, or metastatic chemotherapy [29]. The same year, talazoparib was granted approval by the FDA for patients with deleterious or potentially deleterious gBRCAm, HER-2-negative locally advanced or metastatic breast cancer, given the EMBRACA phase III trial outcomes [30]. The favorable effect on metastatic disease fostered the research of PARP inhibitors also in the early-stage disease. Talazoparib, specifically, is deemed the most potent PARP inhibitor candidate for neoadjuvant single-agent treatment [31]. Additional PARP inhibitors for breast cancer, such as niraparib, rucaparib, and veliparib, are still tested in the clinical setting either as monotherapy and as combinatory options [32]. 


\section{PD-L1 protein - immune checkpoint inhibitors}

PD-L1 is frequently expressed in one out of five patients with TNBC and has been associated with distinct features of breast cancer, such as younger age, large tumor size, high grade, and significant proliferation. PD-L1 is expressed on approximately $10 \%$ of cancer cells and up to $65 \%$ of tumor-infiltrating immune cells(IC) [33]. The immunotherapy agent atezolizumab, marketed as Tecentriq, combined with the immunotherapy regimen albumin-bound paclitaxel or nab-paclitaxel, branded as Abraxane, received approval for the treatment of unresectable locally advanced or metastatic triple-negative, PD-L1-positive breast cancer [28].

The employment of immune checkpoint inhibitors for the stimulation of the immune cells and the tumor growth limitation plays a key role in enhancing the treatment of TNBC. Atezolizumab, an anti-PD-L1 inhibitor, has been the first of such regimens to receive the FDA accelerated approval in March 2019. The approval was obtained due to the sustained efficacy it provided ( 25 months vs. 18 months) as an adjuvant to nab-paclitaxel compared to nab-paclitaxel alone in patients with metastatic TNBC and IC PD-L1 expression (PD-L1+ IC ₹ 1\%) (IMpassion 130 trial) [34]. Moreover, in November 2020, based on the KEYNOTE-355 trial, the FDA granted accelerated approval to pembrolizumab (Keytruda) as an adjuvant to chemotherapy for the management of locally relapsed unresectable or metastatic TNBC with positive PD-L1 expression [35]. Finally, durvalumab (Imfinzi) combined with neoadjuvant anthracycline and taxane-based chemotherapy substantially improved survival rates in patients with early TNBC, according to GeparNUEVO phase II trial, as announced in the 2021 American Society of Clinical Oncology (ASCO) Annual Meeting. The findings of the trial showed that patients in the durvalumab arm obtained a complete response of $53.4 \%$ compared to $44.2 \%$ of patients in the placebo arm [36].

\section{Antibody-drug conjugate targeting TROP-2 - sacituzumab govitecan}

Sacituzumab govitecan is an antibody-drug conjugate comprised of an antibody against an antigen expressed in most types of breast cancer, the human trophoblast cell-surface antigen 2 (TROP-2), paired with topoisomerase I inhibitor (SN-38) via a patented hydrolyzable linker [37]. On April 7, 2021, the FDA approved the use of sacituzumab govitecan (Trodelvy) for patients with unresectable locally advanced or metastatic TNBC who have received previously two or more systemic treatments, at least one of them for metastatic cancer [38].

In the ASCENT phase III clinical trial, a total of 468 patients without brain metastases were randomized to either receive sacituzumab govitecan or chemotherapy. Results showed that the median progression-free survival was nearly six months with sacituzumab govitecan and less than two months with chemotherapy, while sacituzumab govitecan outperformed chemotherapy in the overall survival as well (12.1 months vs. 6.7 months). However, sacituzumab govitecan demonstrated a less satisfactory safety profile regarding treatment-related adverse events of grade 3 or higher than chemotherapy, with neutropenia, leukopenia, diarrhea, anemia, and febrile neutropenia being the most common [37].

\section{Conclusions}

The development of novel regimens for the management of TNBC has demonstrated extraordinary progress over recent years. Unlike in the past, when chemotherapy was the only available option, immunotherapy and targeted inhibitors have been added to the therapeutic armamentarium as part of the treatment approach for advanced TNBC. They are currently under research for the management of early-stage disease too. The ideal sequencing of such regimens is not determined yet and may gradually present a challenge in the real-life clinical setting as further biologic agents will gain approval for TNBC. Finally, a number of additional treatments that explicitly target key pathways of TNBC biology are under development. The development of those agents may alter the TNBC therapeutic landscape in the foreseeable future. Conclusively, these novel lines of research and treatment options will lead to a customized, patient-centered therapeutic approach for both early-stage and advanced TNBC. Treatment decision-making will be guided by specific predictive biomarkers and medications that augment efficacy and improve the safety profile. As a result, the treatment paradigm for TNBC will evolve significantly in the future, improving the survival and patients' quality of life.

\section{Additional Information \\ Disclosures}

Conflicts of interest: In compliance with the ICMJE uniform disclosure form, all authors declare the following: Payment/services info: All authors have declared that no financial support was received from any organization for the submitted work. Financial relationships: All authors have declared that they have no financial relationships at present or within the previous three years with any organizations that might have an interest in the submitted work. Other relationships: All authors have declared that there are no other relationships or activities that could appear to have influenced the submitted work.

\section{References}

1. Siegel RL, Miller KD, Jemal A: Cancer statistics, 2020. CA Cancer J Clin. 2020, 70:7-30. 10.3322/caac.21590 
2. Cancer facts and figures 2021. (2021). https://www.cancer.org/content/dam/cancer-org/research/cancerfacts-and-statistics/annual-cancer-facts-and-figures/20....

3. Hurvitz S, Mead M: Triple-negative breast cancer: advancements in characterization and treatment approach. Curr Opin Obstet Gynecol. 2016, 28:59-69. 10.1097/GCO.0000000000000239

4. Griffiths CL, Olin JL: Triple negative breast cancer: a brief review of its characteristics and treatment options. J Pharm Pract. 2012, 25:319-23. 10.1177/0897190012442062

5. Shaikh SS, Emens LA: Current and emerging biologic therapies for triple negative breast cancer . Expert Opin Biol Ther. 2020, 1-12. 10.1080/14712598.2020.1801627

6. Seligson JM, Patron AM, Berger MJ, Harvey RD, Seligson ND: Sacituzumab govitecan-hziy: an antibody-drug conjugate for the treatment of refractory, metastatic, triple-negative breast cancer. Ann Pharmacother. 2021, 55:921-31. 10.1177/1060028020966548

7. Torre LA, Siegel RL, Ward EM, Jemal A: Global cancer incidence and mortality rates and trends--an update . Cancer Epidemiol Biomarkers Prev. 2016, 25:16-27. 10.1158/1055-9965.EPI-15-0578

8. Prat A, Adamo B, Cheang MC, Anders CK, Carey LA, Perou CM: Molecular characterization of basal-like and non-basal-like triple-negative breast cancer. Oncologist. 2013, 18:123-33. 10.1634/theoncologist.2012-0397

9. Gierach GL, Burke A, Anderson WF: Epidemiology of triple negative breast cancers . Breast Dis. 2011, 32:524. 10.3233/BD-2010-0319

10. Perou CM, Sørlie T, Eisen MB, et al.: Molecular portraits of human breast tumours. Nature. 2000, 406:74752. 10.1038/35021093

11. Sorlie T, Tibshirani R, Parker J, et al.: Repeated observation of breast tumor subtypes in independent gene expression data sets. Proc Natl Acad Sci USA. 2003, 100:8418-23. 10.1073/pnas.0932692100

12. Brenton JD, Carey LA, Ahmed AA, Caldas C: Molecular classification and molecular forecasting of breast cancer: ready for clinical application?. J Clin Oncol. 2005, 23:7350-60. 10.1200/JCO.2005.03.3845

13. Baselga J, Perez EA, Pienkowski T, Bell R: Adjuvant trastuzumab: a milestone in the treatment of HER-2positive early breast cancer. Oncologist. 2006, 11:4-12. 10.1634/theoncologist.11-90001-4

14. Pal SK, Mortimer J: Triple-negative breast cancer: novel therapies and new directions . Maturitas. 2009, 63:269-74. 10.1016/j.maturitas.2009.06.010

15. Sandhu R, Parker JS, Jones WD, Livasy CA, Coleman WB: Microarray-based gene expression profiling for molecular classification of breast cancer and identification of new targets for therapy. Lab Med. 2010, 41:364-72. 10.1309/LMLIK0VIE3CJK0WD

16. Lehmann BD, Jovanović B, Chen X, et al.: Refinement of triple-negative breast cancer molecular subtypes: implications for neoadjuvant chemotherapy selection. PLoS One. 2016, 11:e0157368. 10.1371/journal.pone.0157368

17. Foulkes WD, Smith IE, Reis-Filho JS: Triple-negative breast cancer. N Engl J Med. 2010, 363:1938-48. 10.1056/NEJMra1001389

18. Liedtke C, Mazouni C, Hess KR, et al.: Response to neoadjuvant therapy and long-term survival in patients with triple-negative breast cancer. J Clin Oncol. 2008, 26:1275-81. 10.1200/JCO.2007.14.4147

19. Almendro V, Cheng YK, Randles A, et al.: Inference of tumor evolution during chemotherapy by computational modeling and in situ analysis of genetic and phenotypic cellular diversity. Cell Rep. 2014, 6:514-27. 10.1016/j.celrep.2013.12.041

20. Balko JM, Giltnane JM, Wang K, et al.: Molecular profiling of the residual disease of triple-negative breast cancers after neoadjuvant chemotherapy identifies actionable therapeutic targets. Cancer Discov. 2014, 4:232-45. 10.1158/2159-8290.CD-13-0286

21. Wang Y, Waters J, Leung ML, et al.: Clonal evolution in breast cancer revealed by single nucleus genome sequencing. Nature. 2014, 512:155-60. 10.1038/nature13600

22. Islam S, Zeisel A, Joost S, et al.: Quantitative single-cell RNA-seq with unique molecular identifiers. Nat Methods. 2014, 11:163-6. 10.1038/nmeth.2772

23. Gupta GK, Collier AL, Lee D, et al.: Perspectives on triple-negative breast cancer: current treatment strategies, unmet needs, and potential targets for future therapies. Cancers (Basel). 2020, 12:2392. 10.3390/cancers 12092392

24. Sharma P, Klemp JR, Kimler BF, et al.: Germline BRCA mutation evaluation in a prospective triple-negative breast cancer registry: implications for hereditary breast and/or ovarian cancer syndrome testing. Breast Cancer Res Treat. 2014, 145:707-14. 10.1007/s10549-014-2980-0

25. Prakash R, Zhang Y, Feng W, Jasin M: Homologous recombination and human health: the roles of BRCA1, BRCA2, and associated proteins. Cold Spring Harb Perspect Biol. 2015, 7:a016600. 10.1101/cshperspect.a016600

26. Audebert M, Salles B, Calsou P: Involvement of poly(ADP-ribose) polymerase-1 and XRCC1/DNA ligase III in an alternative route for DNA double-strand breaks rejoining. J Biol Chem. 2004, 279:55117-26. 10.1074/jbc.M404524200

27. Dhillon KK, Swisher EM, Taniguchi T: Secondary mutations of BRCA1/2 and drug resistance. Cancer Sci. 2011, 102:663-9. 10.1111/j.1349-7006.2010.01840.x

28. Triple-negative breast cancer. (2021). https://www.breastcancer.org/symptoms/types/triple-negative.

29. Robson M, Ruddy KJ, Im SA, et al.: Patient-reported outcomes in patients with a germline BRCA mutation and HER2-negative metastatic breast cancer receiving olaparib versus chemotherapy in the OlympiAD trial. Eur J Cancer. 2019, 120:20-30. 10.1016/j.ejca.2019.06.023

30. Ettl J, Quek RG, Lee KH, et al.: Quality of life with talazoparib versus physician's choice of chemotherapy in patients with advanced breast cancer and germline BRCA1/2 mutation: patient-reported outcomes from the EMBRACA phase III trial. Ann Oncol. 2018, 29:1939-47. 10.1093/annonc/mdy257

31. Litton JK, Scoggins M, Ramirez DL, et al.: A feasibility study of neoadjuvant talazoparib for operable breast cancer patients with a germline BRCA mutation demonstrates marked activity. NPJ Breast Cancer. 2017, 3:49. 10.1038/s41523-017-0052-4

32. Cocco S, Piezzo M, Calabrese A, et al.: Biomarkers in triple-negative breast cancer: state-of-the-art and future perspectives. Int J Mol Sci. 2020, 21:4579. 10.3390/ijms21134579

33. Van Berckelaer C, Rypens C, van Dam P, et al.: Infiltrating stromal immune cells in inflammatory breast 


\section{Cureus}

cancer are associated with an improved outcome and increased PD-L1 expression. Breast Cancer Res. 2019, 21:28. 10.1186/s13058-019-1108-1

34. Schmid P, Adams S, Rugo HS, et al.: Atezolizumab and nab-paclitaxel in advanced triple-negative breast cancer. N Engl J Med. 2018, 379:2108-21. 10.1056/NEJMoa1809615

35. FDA grants accelerated approval to pembrolizumab for locally recurrent unresectable or metastatic triplenegative breast cancer. (2020). https://www.fda.gov/drugs/drug-approvals-and-databases/fda-grantsaccelerated-approval-pembrolizumab-locally-recurren....

36. Loibl S, Schneeweiss A, Huober J, et al.: Durvalumab improves long-term outcome in TNBC: results from the phase II randomized GeparNUEVO study investigating neodjuvant durvalumab in addition to an anthracycline/taxane based neoadjuvant chemotherapy in early triple-negative breast cancer (TNBC). J Clin Oncol. 2021, 39:506. 10.1200/JCO.2021.39.15_suppl.506

37. Bardia A, Hurvitz SA, Tolaney SM, et al.: Sacituzumab govitecan in metastatic triple-negative breast cancer . N Engl J Med. 2021, 384:1529-41. 10.1056/NEJMoa2028485

38. FDA grants regular approval to sacituzumab govitecan for triple-negative breast cancer . (2021). https://www.fda.gov/drugs/resources-information-approved-drugs/fda-grants-regular-approvalsacituzumab-govitecan-trip... 